\title{
Change of practice patterns in urology with the introduction of the Da Vinci surgical system: The Greek NHS experience in debt crisis era
}

\author{
Dimitros Deligiannis, Ioannis Anastasiou, Vasileios Mygdalis, \\ Evangelos Fragkiadis. Konstantinos Stravodimos \\ $1^{\text {st }}$ Department of Urology, University of Athens, Medical School "Laiko" Hospital, Athens, Greece.
}

\begin{abstract}
Summary Objective: To determine the attitudinal change for urologic surgery in Greece since the introduction of the da Vinci Surgical System (DVS). We describe contemporary trends at public hospital level, the initial Greek experience, while at the same time Greece is in economic crisis and funding is under austerity measures. Materials and Methods: We retrospectively analyzed annualized case log data on urologic procedures, between 2008 (installation of the DVS) and 2013, from "Laiko" Hospital in Athens. We evaluated, using summary statistics, trends and institutional status regarding robot-assisted surgery (RAS). We also analyzed the relationship between the introduction of RAS and change in total volume of procedures performed. Results: 1578 of the urological procedures performed at "Laiko" Hospital were pooled, 1342 (85\%) being open and 236 RAS (15\%). We observed a 6-fold increase in the number of RAS performed, from $7 \%$ of the total procedural volume $(14 / 212)$ in 2008 to $30 \%(96 / 331)$ in 2013. For radical prostatectomy, in $20082 \%$ were robot-assisted and 98\% open while in 2013, $46 \%$ and $54 \%$ respectively. Pyeloplasty was performed more often using the robot-assisted method since 2010. RAS-dedicated surgeons increased both RAS and the total number of procedures they performed. From 86 in 2008 to 145 in 2013, with 57\% of them being RAS in 2013 as compared to $13 \%$ in 2008.

Conclusions: Robot-assisted surgery has integrated into the armamentarium for urologic surgery in Greece at public hospital level. Surgical robot acquisition is also associated with increased volume of procedures, especially prostatectomy, despite the ongoing debate over cost-effectiveness, during economic crisis and International Monetary Fund (IFN) era.
\end{abstract}

KeY WORDS: Urology in Greece; Da Vinci; Practice patterns; Prostatectomy; Robot-assisted surgery.

Submitted 13 October 2014; Accepted 30 November 2014

\section{INTRODUCTION}

There is increasing patient and surgeon interest in minimally-invasive techniques, particularly with the introduction of the Da Vinci Surgical System in 2001. The majority of procedures carried out are urological, with cardiac, gynecological and general surgeons also using the DVS in practice (1). Since the first reports, RAS has become a fundamental part of urologic surgery with initial enthusiasm changing to a broad acceptance (22). There is an increasing adoption of RAS with robot-assisted radical prostatectomy (RARP) being the most commonly carried out robotic procedure worldwide, with increasing numbers performed each year $(1,5)$.

Robot-assisted radical nephrectomy (RAN) is showing also an increasing development (2). The robot has also allowed non-experienced surgeons to successfully perform robotassisted pyeloplasty (RPyel) with excellent results $(3,4)$. Other procedures also performed are partial nephrectomy and cystectomy.

There is limited knowledge about how the diffusion of robotics has influenced urological individual practice patterns in Greece. The first Da Vinci Surgical System (DVS) in a public hospital in Greece was installed in July of 2008 at "Laiko" Hospital in Athens. There are seven more installed in Greece, all based in private hospitals. Most procedures currently being carried out are urological with general surgeons also using the system, mostly for bariatric procedures. Urologists have been quick to embrace this novel technology and RARP is the most commonly carried out robotic procedure, followed by RPyel, RAN and robot-assisted nephro-ureterectomy (RNUT). This is extremely interesting taking into account the fact that Greece is suffering from economic crisis and funding is under austerity measures proposed by the International Monetary Fund (IMF) (23). Using retrospective case log data from "Laiko" Hospital, in this study we seek to determine practice patterns in open and robotic urologic surgery in Greece since the installation of the DVS.

This study examined contemporary trends in a public hospital in Greece and the relationship between robot acquisition and changes in the total procedural volume.

\section{Materials and Methods}

Data

For our study we obtained annualized case log data on urologic procedures between 2008, when the DVS was 
installed, and 2013, from "Laiko" Hospital in Athens, Greece. We identified six urologic surgeons who performed the procedures, two of whom were RAS-dedicated surgeons with specialized training. The procedures that were pooled and analyzed, open and robot-assisted respectively, are: prostatectomy, nephrectomy, nephroureterectomy and pyeloplasty. Patient characteristics were not recorded or analyzed at this instance.

\section{Methods}

We used summary statistics to describe current practice patterns, after stratifying the surgical procedures with regard to the following variables: a) The surgeon who performed the procedure (dedicated RAS or not); b) Open or robot-assisted surgery; c) Type of procedure: prostatectomy, nephrectomy, nephro-ureterectomy and pyeloplasty. We analyzed trends in open and RAS in each type of procedure.

Especially in prostatectomy and pyeloplasty, where a large attitudinal change seemed to exist. We also analyzed trends regarding each surgeon separately, especially the two RAS-dedicated surgeons.

Furthermore, we determined the relationship between the introduction of RAS and change in the total volume of procedures performed at "Laiko" Hospital.

\section{RESULTS}

1578 of the urological procedures that were performed at "Laiko" Hospital in Athens, Greece, between 2008 and 2013, of whom 1342 (85\%) were open and 236 RAS (15\%), were pooled and studied.

These procedures were prostatectomy, open and RAS (RRPRARP), nephrectomy, open and RAS (ORN-RAN), nephroureterectomy, open and RAS (ONUTRALNU) and pyeloplasty, open and RAS (OPyel-RLPP), respectively. We identified six surgeons. Surgeons A and B, were RASdedicated surgeons, surgeons C and $\mathrm{D}$ performed exclusively open procedures and the other two, surgeons $\mathrm{E}$ and F, performed mostly open procedures and rather occasionally RAS.

Surgeons A and B were 50 and 48 years old respectively, having specialized training in laparoscopy and urologic robotics.

They performed 199 RAS of a total of 236 robot-assisted procedures between 2008 and 2013 (84\%).

Surgeons $C$ and D were 57 and 56 years old respectively, without specialized training and performed exclusively open procedures.

Surgeons $\mathrm{E}$ and $\mathrm{F}$ were 59 and 45 years old respectively and performed the remaining 37 RAS (16\%), having basic training in robotics and having the RAS-dedicated surgeons as actors-proctors. A large increase in the number of urological procedures performed with robotassisted approach during the 6-year study period was recorded. In 2008, urologists performed 198 open pro-

\section{Figure 1.}

cedures and 14 RAS, which is $7 \%$ of the total procedural volume. This percentage gradually increased to $9 \%$ in $2009,11 \%$ in $2010,18 \%$ in 2012. In 2013, which was the last year studied, 235 open procedures and 96 RAS were performed, with RAS being $30 \%$ of the total procedural volume. There was an almost 6 -fold increase in RAS from 2008 to 2013, but also an increase by 56\% in the total procedural volume.

However, we observed that there was a clear downward trend in RAS in 2010-2011 that is attributed to the beginning of the dept crisis era in Greece.

In fact, the DVS was not operating for a major period from 2010 to 2011, due to lack of funds for yearly maintenance and supply of disposal instrumentation. Even though this adverse conjuncture, there was a large increase in the number of RAS performed during the 6year study period.

Furthermore, we observed that after the introduction of the DVS at "Laiko" Hospital and especially after 2012 when it was in use again, there was a significant increase in the total number of procedures performed by the six high-volume surgeons, from 212 procedures in 2008 to 331 in 2013.

Figures 1 and 2 show the relationship between open, RAS procedures and the total procedural volume from 2008 to 2013.

\section{Type of procedure}

When stratifying the procedures by type, we observed a noticeable change both in the annual proportion of prostatectomies and pyeloplasties performed using the robot-assisted method and in the total volume, from 2008 to 2013.

Rompared to open and total procedural volume from 2008 to 2013.
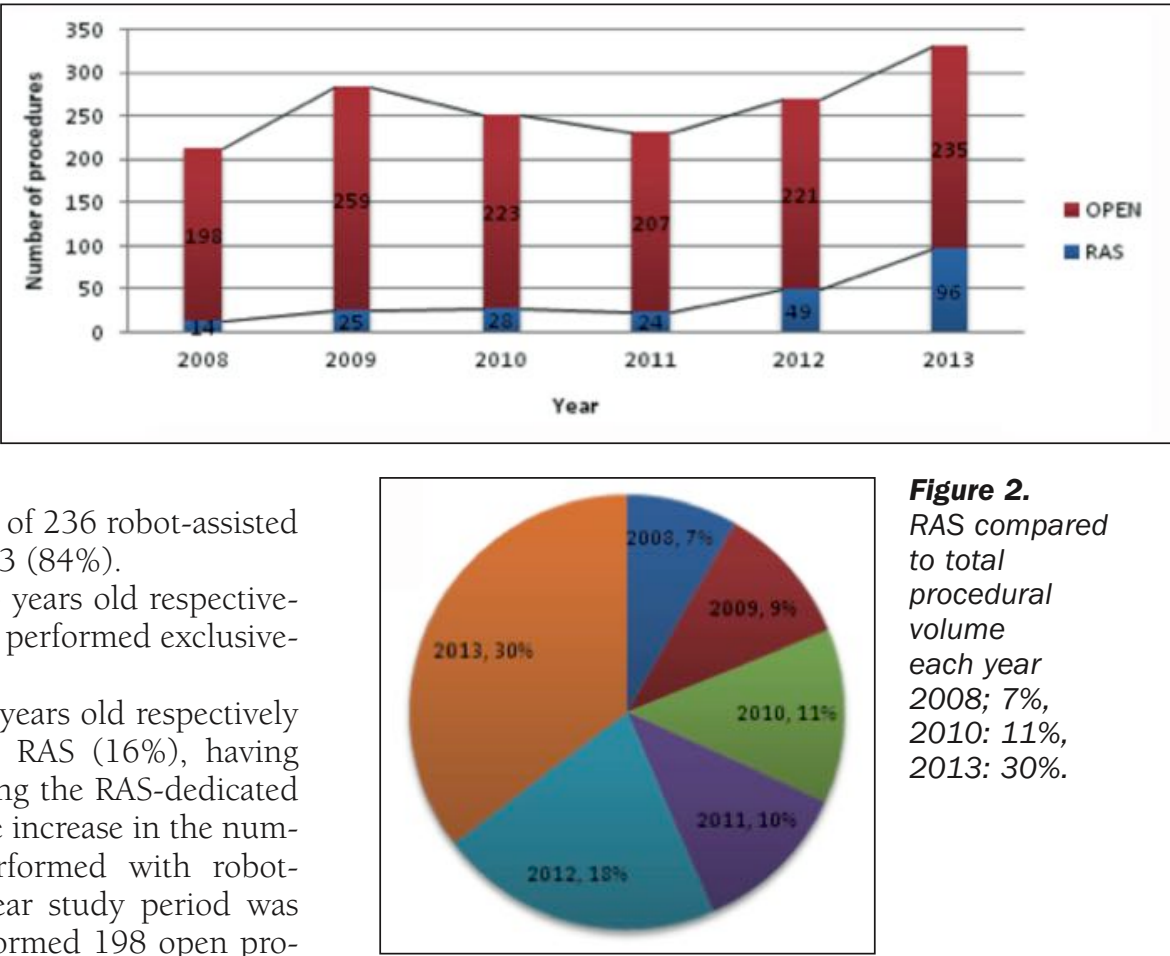

Figure 2.

RAS compared to total procedural volume each year 2008; 7\%, 2010: $11 \%$, 2013: $30 \%$. 
Figure 3.

The evolution of open and robot-assisted radical prostatectomy.

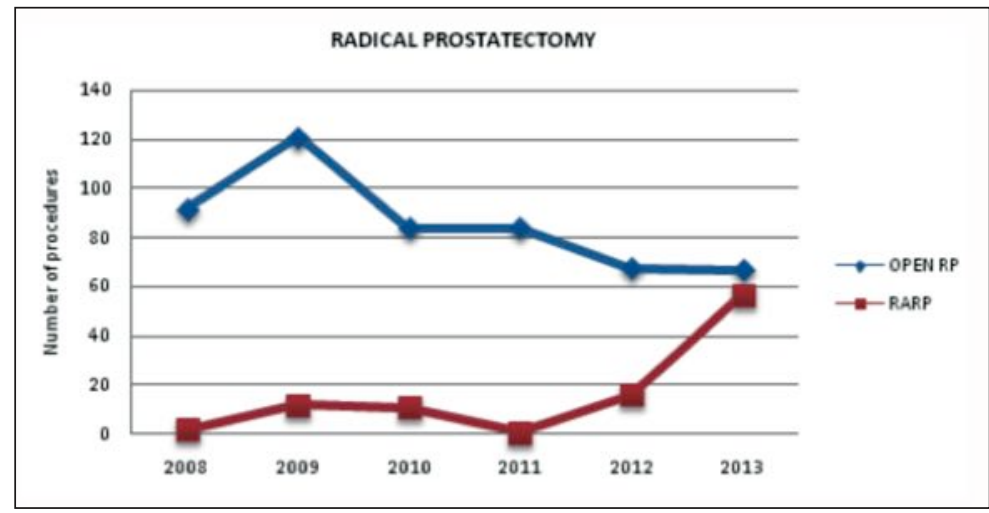

Figure 4.

Radical prostatectomies performed both open and RAS by the six surgeons from 2008 to 2013.

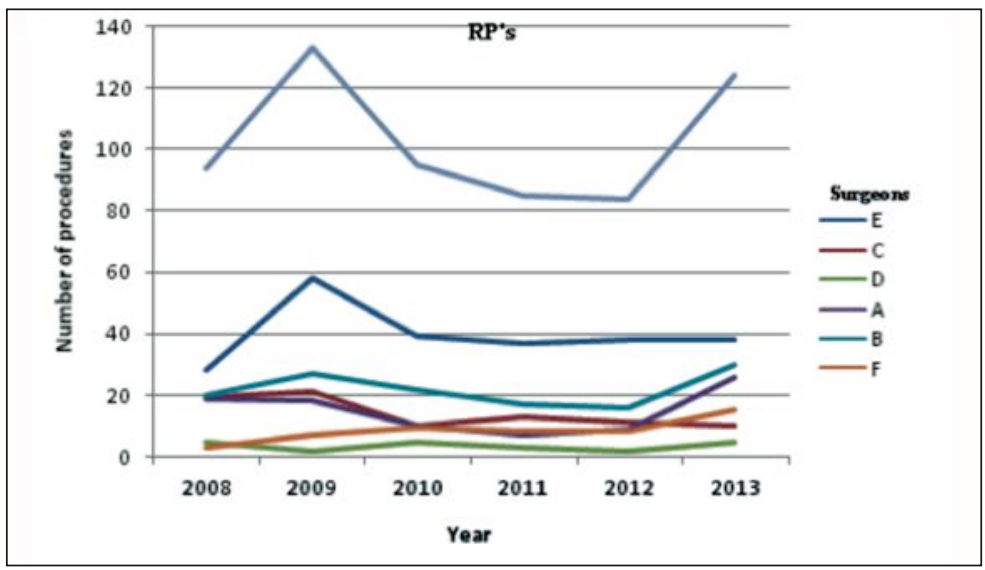

Figure 5.

Evolution in the numbers of open and RAS pyeloplasty.

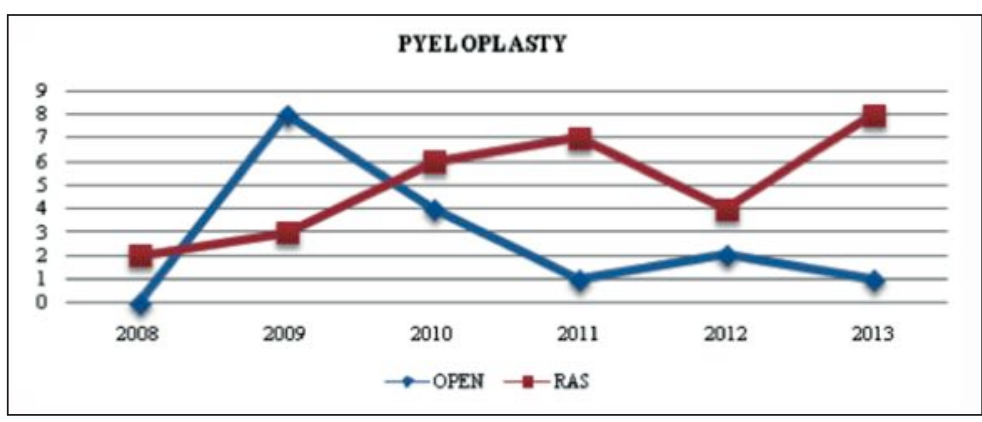

Figure 6.

Procedures done open and RAS by the 2 RAS dedicated surgeons and the comparison with the total procedural volume.

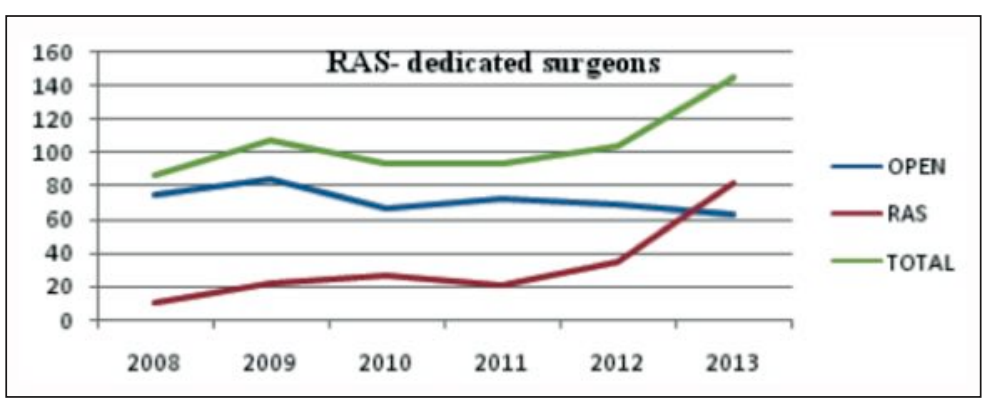

\section{Prostatectomy}

Figure 3 shows the evolution of radical prostatectomy (RP) performed open and robotassisted, from 2008 to 2013. In 2008 urologists in "Laiko" Hospital, performed a total of 94 RP, two of them being RAS (2\%). In 2013 they performed 124 RP, 57 of them being RAS, which is $46 \%$ of the total volume of procedures and 67 open, which is 54\% of the total procedural volume. We observed a dramatic increase both in the total number of RP during the 6-year study period and in the number of RAS. Thus in 2013 there was a 27-fold increase in RARP and the number of RARP was almost equal to that of open RP.

Figure 4 shows, the increase in the total number of RP's performed at "Laiko" Hospital after the introduction of the DVS and especially after 2012, when it was in broad use again.

We recorded that the increase is attributed mostly to the two RAS-dedicated surgeons and consequently to the RARPs they performed, since the other surgeons and especially those performing exclusively open procedures kept almost steady the number of procedures they performed reaching a plateau.

\section{Pyeloplasty}

Figure 5 shows the attitudinal change in pyeloplasty from 2008 to 2013 in "Laiko" Hospital with the use of the DVS. In 2008, only two pyeloplasties were performed, both with the robot-assisted method. In 2010, pyeloplasties were done more often with the robot-assisted method rather than open, while in 2013 eight out of nine procedures performed were robot-assisted (89\%).

\section{RAS - dedicated surgeons}

Figure 6 shows the procedures performed by the two RAS-dedicated surgeons, A and B, from 2008 to 2013. A large increase in the number of RAS done was identified. They gradually decreased the number of open procedures and increased the number of RAS performed and consequently in 2013, they performed more RAS than open procedures. Furthermore, the two RAS-dedicated surgeons increased the total procedural volume performed by them from 86 procedures in 2008 to 145 in 2013, with 13\% of then being RAS in 2008 and $57 \%$ in 2013. The introduction and use of the DVS resulted in more patient seeking treatment at "Laiko" Hospital in order to undergo RAS and consequently as the numbers of RAS increased, the total procedural volume increased also.

The annual proportion of procedures done by surgeon A increased by almost 70\%, with a large increase in the number of RAS, during the 6-year study period. The percentage of RAS cases he performed increased from 13\% in 2008 to $72 \%$ in 2013. At the same time there 
Figure 7.

Surgeons' A practice patterns regarding radical prostatectomy.

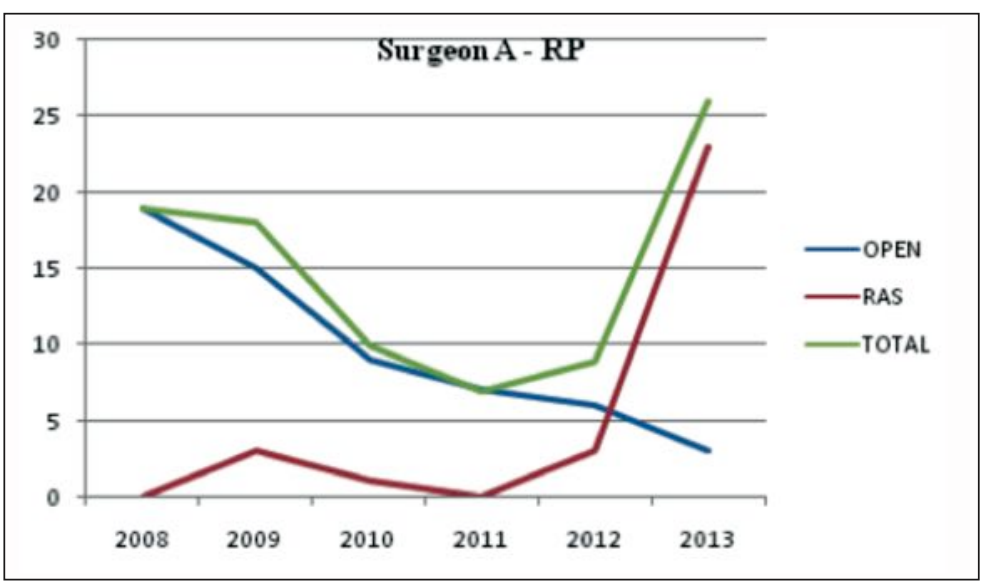

RARP remains the most commonly performed RAS with increasing numbers performed each year. While 1500 RARP were performed in the United States in 2000, that number increased to 8000 in 2004 and 50000 in $2007(2,8)$. In $2010,67 \%$ of RP were done robotically in the U.S. with a substantial increase in the total number of procedures performed (9). The most commonly reported estimates from SEER (Surveillance, Epidemiology and End Results)Medicare are not current, but show that minimally invasive RP rate increased from $9 \%$ in 2003 to $42 \%$ in $2006(9,19)$.

Indeed, most urologists declare RARP to be the gold standard for prostatectomy (2). It has surpassed open RP, despite the lack of prospective evidence showing its oncologic advantages or cost-effectiveness $(9,14)$.

Robot-assisted nephrectomy is a more recent

was a 20-fold increase in the number of RARP, especially after 2012 and an increase in the total volume of radical prostatectomies by 36\%, as Figure 7 shows. Surgeon B, increased his RAS cases from $12 \%$ in 2008 to $39 \%$ in 2013. He decreased the number of open RP, increased the number of RARP by 10 -fold and the total number of RPs by $50 \%$.

\section{Discussion}

Since the FDA clearance for the DVS in prostate surgery was granted in 2001 ( 7,11$)$, urology has seen a dramatic clinical expansion and explosion of RAS and especially its greatest application with RP. Other studies have previously examined practice patterns in minimally invasive surgery in financially-advanced countries: one survey of urologists in the Midwest United States in 2003 regarding laparoscopic surgery $(2,17)$, a study in 2012 in the US about current trends in RARP (9) and a survey in 2014 about urologic laparoscopy in Germany, Austria and Switzerland (18). Thus one of the major advantages of the present study is that it gives us the first results of the use of the DVS, in a country in debt crisis with austerity measures and funding being under the IMF guidance (23).

Comparative studies have suggested RAS to have proven benefits compared to open procedures (3). Most important of them being decreased blood loss, decreased postoperative pain, shorter hospital stay, faster mobilization and faster recovery of the patient and less wound complications. Likewise, similar advantages have been demonstrated for RARP in several studies (11-13). Furthermore, decreased blood loss, fastest recovery of urinary incontinence and more effective nerve-sparing technique, are the most important advantages of RARP (24-26). No difference in terms of oncologic outcomes has been reported $(11,14)$.

The most important drawbacks of the DVS is the substantial cost, its long setup time and mostly longer operation $(2,3,16)$. The robot itself costs about $\$ 1,3-\$ 1,5$ million, with yearly maintenance fee of $\$ 100,000$, and a recurring cost of $\$ 400$ to $\$ 1200$ per case for disposable instrumentation, depending upon the procedure (16). development of robotic urologic surgery (2), but the technical advantages over the standard laparoscopic procedure are less evident than those of RP (10). It is interesting that robot-assisted partial nephrectomy (PN) seems to have now supplanted laparoscopic PN as the most common minimally invasive approach for PN (21). Greek citizens had the opportunity to have free access to the DVS via public health services after the installation of the system at "Laiko" Hospital in Athens, a public hospital, in July 2008. Our analysis of case log data from "Laiko" Hospital provides insight into contemporary practice patterns of Greek urologists, regarding robot-assisted surgery.

The most important study finding is the substantial increase in the number of RAS in this 6-year period. As well, an increase in the total volume of procedures done during the study was also observed. We found that RAS - dedicated urologists who performed RAS and especially RARP had a higher annual volume than those who performed only open procedures. Based on these finding and those of others $(9,19,20)$, it appears that the uptake of robotics has contributed to the centralization of urological procedures (mostly RP) in the hands of higher volume surgeons. Indeed, surgeons who performed RAS in our study had a higher volume and a greater absolute number of procedures. Apart from urologists with specialized training, other surgeons who had basic training in robotics also performed RAS depending probably on the affiliation with the academic hospital, the existence of the DVS and the existing surgical skills, having the RAS-dedicated surgeons as actors-proctors.

Significant factors that contributed to the prevalence of RAS in urology in "Laiko" Hospital, as mentioned by previous similar studies (18), were: use of trained personnel (nurse, anesthesiologist), structured training programs (in cooperation with Karolinska University Hospital, Stockholm, Sweden), dedicated operating room, organized surgery schedule exclusively for RAS. The benefits of RAS for the patients are proven and undeniable, especially for RARP.

There is also increasing interest and desire from the patients to have a robot-assisted surgery after the introduction of the DVS. In order to keep in touch with the 
novel technology and patients' selection for minimally invasive techniques, urologists at "Laiko" Hospital switched towards RAS in line with developed countries worldwide. As a result and due to the broad use of the DVS in urologic surgery, an increasing number of patients are turning to the Greek national health system, seeking treatment mostly for RP. The effort made in "Laiko" Hospital and the results are being highlighted by the fact that over the last years the adverse conjuncture of debt crisis in Greece has led to dramatic changes in health care system (23). It has to be mentioned, that the activity of the DVS was suspended for almost 1 year from 2010 to 2011. Even though, the study suggests that RAS has become a surgical standard in urology in the Greek national health system (NHS), especially for procedures such as radical prostatectomy, where the benefits are proven and undeniable $(3,15)$. Due to austerity measures, a very few resources were available for public health in Greece. On the other hand, surgeons at "Laiko" Hospital had to be consistent with the university setting and the educational element of the Hospital. So, despite cost cuts they transferred funds in order to keep in touch with technology and sustain in use the DVS. A limitation of this study is its retrospective design. Moreover it is a purely descriptive study, lacking any clinical or pathological data that would provide valuable clinical information. Selection bias could be considered the specific types of procedures pooled and examined, not including the whole armamentarium of urologic surgery.

The number of procedures done open and RAS are rather small. However, this study highlights the development of RAS in a public hospital in a country at dept crisis. Therefore it could be a stepping stone for a cost-effectiveness analysis of the DVS in the context of public National Health System hospitals.

\section{Conclusions}

Robot- assisted surgery using the DVS has integrated into the minimally invasive armamentarium for urologic surgery in Greece at national hospital level, in line with developed countries worldwide. Its greatest application is seen in radical prostatectomy and pyeloplasty. There is attitudinal change towards RAS, which is combined with an increase in the total volume of procedures performed, which is mostly attributed to the experience gradually gained, the undeniable benefits for the patients and patients' desire. Surgeon characteristics and practice patterns have a clear role in the type of the procedure performed, as RAS-dedicated surgeons have dramatically increased both the number of RAS and the total procedural volume performed. The significant recession and debt crisis in Greece this period highlights the value of this study, showing the increasing use of RAS despite the ongoing debate over its usefulness and cost-effectiveness, in a country with strict austerity measures.

\section{REFERENCES}

1. Murphy DG1, Hall R, Tong $R$, et al. Robotic technology in surgery: current status in 2008. ANZ J Surg. 2008; 78:1076-1081
2. Yuh BE, Hussain A, Chandrasekhar R, et al. Comparative analysis of global practice patterns in urologic robot-assisted surgery. $J$ Endourol. 2010; 24:1637-1644.

3. Thiel DD, Winfield HN. Robotics in urology: past, present and future. J Endourol. 2008; 22:825-30.

4. Bentas W, Wolfram M, Brautigam R, et al. Da Vinci robot assisted Anderson-Hynes dismembered pyeloplstay: Technique and 1 year follow-up. World J Urol. 2003; 21:133.

5. Intuitive Surgical. Intuitive Surgical Investor Report 2007. Available from URL: http://investor.intuitivesurgical.com/phoenix. $z$ html?c=122359\&p=irol-IRHome (accessed 5 January 2008).

6. Davies BL, Hibberd RD, Copcoat MH, Wickham JE. A surgeon robot prostatectomy-a laboratory evaluation. J Med EngTechnol. 1989; 13:273.

7. Davies BL, Hibberd RD, Ng Ws, et al. The development of a surgeon robot for prostatectomies. ProcInst Mech Eng. 1991; 205:35.

8. WexnerSd, Bergamashi R, Lacy A, et al. The current status of robotic pelvic surgery: Results of a multinational inter-disciplinary consensus conference. Surg Endosc. 2009; 23:438-443.

9. Lowrance WT, Eastham JA, Savage A, et al. Contemporary open and robotic radical prostatectomy practice patterns among urologists in the United States. J Urol. 2012; 187:2087-2092.

10. Poon SA, Silberstein JL, Chen LY, et al. Trend in partial and radical nephrectomy: An analysis of case logs from certifying urologists. Journal of Urology. 2013; 190:464-469.

11. Subhasis G, Diptendra KS. Current status of robotic surgery. Indian J Surg. 2012; 74:242-247.

12. AhleringTe, Woo D, Eichel L, et al. Robot-assisted versus open radical prostatectomy: a comparison of one surgeon's outcomes. Urology. 2004; 63:819-822.

13. Menon M, Tewari A, Baise B, et al. Prospective comparison of radical retropubic prostatectomy and robot-assisted anatomic prostatectomy: the Vattikuti Urology Institute experience. Urology. 2002; 60:864-868.

14. Ficarra V, Novara G, Fracalanza S, et al. A prospective, nonrandomized trial comparing robot-assisted laparoscopic and retropubic radical prostatectomy in one European Institution, BJU Int. 2009; 104:534-539.

15. Farnham SB, Webster TM, Herrell SD, Smith JA. Intraoperative blood loss and transfusion requirements for robotic-assisted radical prostatectomy. Urology. 2006; 67:360

16. Thaly R, Patel VR, Shah KK. The robotic revolution: Advancing laparoscopy nad urology further into the future. Contemp Urol. 2006; 18:28.

17. Wang DS, Winfield HN. Survey of urological laparoscopic practice patterns in the Midwest. J Urol. 2004; 172:2282-2286.

18. Imkamp F, Herrmann TRW, Stolzenburg JU, et al. Development of urologic laparoscopy in Germany, Austria, and Switzerland: a survey among urologists. World J Urol. 2014;DOI 10.1007/s00345014-1250-4

19. Makarov DV, Yu JB, Desai RA, et al. The association between diffusion of the surgical robot and radical prostatectomy rates. Med Care. 2011; 49:333.

20. Stitzenberg KB, Wong YN, Nielsen ME, et al. Trends in radical prostatectomy: centralization, robotics, and access to urologic cancer care. Cancer. 2011; 118:54. 
21. Ghani KR, Sukumar S, Sammon JD, et al. Practice patterns and outcomes of open and minimally invasive partial nephrectomy since the introduction of robotic partial nephrectomy: Results from the nationwide inpatient sample. J Urol. 2014; 191:907-913.

22. Guru KA, Hussain A, Chandrasekhar R, Current status of robotassisted surgery in urology: a multi-national survey of 297 urologic surgeons. Can J Urol. 2009; 16:4736-41.

23. Kentikelenis A, Karanikolos M, Reevers A, Greece's health crisis: from austerity to denialism. The Lancet. 2014; 383:748-753.
24. Novara G, Ficarra V, Wilson TG. Systematic review and metaanalysis of perioperative outcomes and complications after robotassisted radical prostatectomy Eur Urol. 2012; 62:431-52.

25. Ficarra V, Novara G, Rosen RC. Systematic review and metaanalysis of studies reporting urinary continence recovery after robotassisted radical prostatectomy Eur Urol. 2012; 62:405-17.

26. Ficarra V, Novara G, Montorsi F. Systematic review and metaanalysis of studies reporting potency rates after robot-assisted radical prostatectomy Eur Urol. 2012; 62:418-30.

\section{Correspondence}

Dimitros Deligiannis, MD (Corresponding Author)

d.delijohn@yahoo.gr

Ioannis Anastasiou, MD

ekati2@otenet.gr

Vasileios Mygdalis, MD

vasmig@gmail.com

Evangelos Fragkiadis, MD

frangiadis@yahoo.com

Konstantinos Stravodimos, MD

kgstravod@yahoo.com

$1^{\text {st }}$ Department of Urology, University of Athens, Medical School

"Laiko" Hospital, 17 Agiou Thoma str., 11527 Athens (Greece) 